\title{
Superior mesenteric vein thrombosis: A rare cause of painless diarrhea
}

\author{
Adam Zayac ${ }^{1 *}$, Komal Akhtar ${ }^{1}$, Arundeep Kahlon ${ }^{1}$, Jalaluddin Umar ${ }^{2}$, Viveksandeep Thoguluva Chandrasekar ${ }^{2}$ and Tanya George ${ }^{2}$ \\ ${ }^{1}$ Department of Internal Medicine, SUNY Upstate Medical University Hospital, 750 E Adams St, Syracuse, USA \\ ${ }^{2}$ Division of Hematology-Oncology, SUNY Upstate Medical University Hospital, 750 E Adams St, Syracuse, USA
}

\begin{abstract}
Superior Mesenteric Vein (SMV) Thrombosis is a rare disease entity. Patients can develop life-threatening complications including hemorrhagic shock or intestinal infarction, but if it is found early, it is treatable and potentially reversible. We present a case of a 56-year-old woman admitted with chronic diarrhea, who was found to have non-occlusive SMV thrombosis.

A 56-year-old female presented to our hospital with complaints of fever and diarrhea. She had been recently diagnosed with Diffuse B-Cell Lymphoma and was on Rituximab, Cyclophosphamide, Doxorubicin, Vincristine and Prednisone (R-CHOP) chemotherapy; her last dose was two weeks prior to admission. Four weeks earlier, she developed non-bloody, non-foul smelling diarrhea without abdominal pain. Her diarrhea did not improve, even after discontinuing all medications with this potential side effect. She had an unremarkable upper endoscopy. Infectious workup including stool studies was unrevealing. She did not improve with empiric antibiotic therapy. A computerized tomography (CT) scan of the abdomen and pelvis showed decreased lymphadenopathy, and a new SMV thrombus. She was started on anticoagulation therapy with enoxaparin and her symptoms improved in next few days, with complete resolution after two weeks.

SMV Thrombosis is a rare condition that can cause an array of symptoms, including diarrhea. The mechanism by which it causes diarrhea is thought to be related to ion channel dysfunction and secretory diarrhea. SMV Thrombosis can be treated surgically or with anticoagulation. If left untreated, it can progress to bowel infarction and death. For this reason, it should be considered in patients with chronic diarrhea with a hypercoagulable state and should be evaluated with appropriate imaging.
\end{abstract}

\section{Background}

Superior Mesenteric Vein (SMV) thrombosis is a rare disease entity that can result in life-threatening complications including hemorrhagic shock or intestinal infarction, but if discovered early, is treatable and potentially reversible. We present a case of a 56-year-old woman admitted with chronic diarrhea, who was found to have nonocclusive SMV thrombosis.

\section{Case}

A 56-year-old female presented to our hospital with complaints of fever and diarrhea. Her past medical history included type 1 diabetes mellitus, end-stage renal disease (ESRD), renal and pancreas transplant with subsequent pancreatectomy, requiring enzyme replacement. She was receiving Rituximab, Cyclophosphamide, Doxorubicin, Vincristine and Prednisone (R-CHOP) chemotherapy for recently diagnosed Diffuse B-Cell Lymphoma, with her last dose two weeks prior to presentation. Four weeks earlier, she developed non-bloody, nonfoul smelling diarrhea without abdominal pain and developed fever one week prior to admission. A recent positron emission tomography (PET) scan showed significant intra-abdominal and retroperitoneal lymphadenopathy, without uptake in the gastrointestinal tract and an unremarkable upper endoscopy. All medications with the potential to cause diarrhea were discontinued without improvement. Upon admission, she was afebrile with a benign abdominal exam. Systemic infectious workup was unrevealing. Stool studies including clostridium difficile PCR, ova and parasites, gram stain, fecal fat, stool $\mathrm{pH}$, and stool cultures were also negative. She did not improve with empiric antibiotic therapy. A computerized tomography (CT) scan of the abdomen and pelvis showed decreased lymphadenopathy and a Superior Mesenteric Vein thrombosis. She was started on therapeutic anticoagulation; her symptoms improved with complete resolution of diarrhea after two weeks.

\section{Discussion}

SMV Thrombosis is a rare condition that can cause an array of symptoms, from severe pain to diarrhea [1]. The mechanism by which it causes diarrhea is thought to be related to bowel wall edema leading to vascular congestion from impaired venous drainage. In turn, this causes ion channel dysfunction and secretory diarrhea $[2,3]$.

SMV Thrombosis can be treated surgically or with anticoagulation. However, with improved imaging techniques and increasing use of anticoagulation the prognosis of this condition has improved $[1,3,4]$. While mesenteric arterial thrombosis results from arrhythmia and cardiac etiologies, mesenteric venous thrombosis is overwhelmingly associated with hypercoagulable states, stasis and local factors which lead to vessel wall injury [3]. These include both inherited

Correspondence to: Adam Zayac, Department of Internal Medicine, SUNY Upstate Medical University Hospital, 750 E Adams St, Syracuse, USA, Tel: $315-$ 464-5540; E-mail: upstateaz@gmail.com

Key words: mesenteric venous thrombosis, diarrhea, hypercoaguable

Received: June 09, 2017; Accepted: June 24, 2017; Published: June 26, 2017 
and acquired hypercoagulable conditions, including patients taking oral contraceptives, pregnant or postpartum women, or those with malignancies [5]. If left untreated, it can progress to bowel infarction and death, with a mortality rate ranging from 20 to $50 \%[5,6]$. Mesenteric venous thrombosis is found in $0.2 \%$ to $2 \%$ of patients at autopsy and due to its indescript symptoms, remains difficult to diagnose [1]. Our case highlights the importance of having a high index of suspicion for this disease entity, as early diagnosis and treatment may prevent the devastating sequelae associated with prolonged thrombosis.

\section{Conclusion}

Evaluation for mesenteric venous thrombosis with appropriate abdominal imaging is pivotal to in patients with known hypercoagulable states, such as those with malignancy, after ruling out infectious etiologies in patients with vague abdominal symptoms. Delay in diagnosis and management can lead to increased morbidity and mortality in these complex patients.

\section{References}

1. Morasch MD, Ebaugh JL, Chiou AC, Matsumura JS, Pearce WH, et al. (2001) Mesenteric venous thrombosis: A changing clinical entity. J Vasc Surg 34: 680-684. [Crossref]

2. https://www.uptodate.com/contents/mesenteric-venous-thrombosis-in-adults

3. Singal AK, Kamath PS, Tefferi A (2013) Mesenteric Venous Thrombosis. Mayo Clinic Proceedings 88: 285-294.

4. Kumar S, Kamath PS (2003) Acute superior mesenteric venous thrombosis: one disease or two? Am J Gastroenterol 98: 1299-1304. [Crossref]

5. Gul W, Markert RJ, Abbass K (2011) Acute Superior Mesenteric Vein Thrombosis: A Lethal Disease. Pract Gastroenterol : 4.

6. Kumar S, Sarr MG, Kamath PS (2001) Mesenteric Venous Thrombosis. N Engl J Med 345: 1683-1588.[Crossref]

Copyright: @2017 Zayac A. This is an open-access article distributed under the terms of the Creative Commons Attribution License, which permits unrestricted use, distribution, and reproduction in any medium, provided the original author and source are credited. 